\title{
Modelo de estimación estadística «Programa Inclusión Productiva» MIPRO-Ecuador
}

\section{Model of statistical estimation «Program of Productive Inclusion» MIPRO-Ecuador}

Juan C. Escobar Sailema es docente en la Escuela de Formación de Soldados del Ejército (Ecuador) e investigador independiente en B\&B, asesoramiento de negocios. (juan.escobar145@gmail.com) (https://orcid.org/0000-0002-5076-1129).

\begin{abstract}
Resumen
La presente investigación demuestra la incidencia de estimaciones de demanda sobre la rentabilidad de los emprendimientos asesorados dentro del programa de «Inclusión Productiva» que lleva a cabo el Ministerio de Industrias y Productividad-Mipro (Ecuador). El aporte académico-práctico que derive del presente objeto de estudio serán insumos para dicho Ministerio, sus respectivas zonales, direcciones y para quienes den uso adecuado del producto final. El objetivo de este trabajo es determinar la incidencia de un modelo de estimación estadística de la demanda en el mejoramiento de la rentabilidad de los emprendimientos, lo que incluye evaluar el modelo de estimación de la demanda empleado, determinar los niveles de rentabilidad, las variables de incidencia, e identificar los componentes de un modelo de estimación estadística. Dichos objetivos fueron alcanzados mediante la recolección de información, medición numérica y el análisis estadístico de los datos. La población objeto de estudio está comprendida por 702 emprendimientos, clasificados en 4 sectores productivos: comercio de bienes, comercio de servicios, manufactura y agropecuario. Estableciendo una muestra de 248 observaciones con un 95\% de nivel de confianza y una desviación estándar 0,5, el resultado obtenido es el diseño de un modelo de estimación estadística de la demanda y la aplicabilidad de este, cuya precisión es totalmente aceptable estadística y financieramente, lo que significa el mejoramiento de la rentabilidad de los emprendimientos asesorados y el cumplimiento de los objetivos institucionales.
\end{abstract}

\begin{abstract}
This research demonstrates the incidence of demand estimates on the profitability of the enterprises advised within the "Productive Inclusion" program carried out by the Ministry of Industries and Productivity-Mipro (Ecuador). The academic-practical contribution that derives from the present object of study will be inputs for this Ministry, their respective zones, addresses and for those who give adequate use of the final product. The objective of this work is to determine the incidence of a model of statistical estimation of the demand in the improvement of the profitability of the enterprises, which includes evaluating the model of estimation of the used demand, to determine the levels of profitability, the variables of incidence, and to identify the components of a model of statistical estimation. These objectives were achieved through data collection, numerical measurement, and statistical analysis of the data. The target population is comprised of 702 enterprises, classified into 4 productive sectors: trade in goods, trade in services, manufacturing and agriculture. Establishing a sample of 248 observations with a $95 \%$ confidence level and a standard deviation of 0.5, the result obtained is the design of a model of statistical estimation of the demand and its applicability, whose precision is statistically and financially acceptable, which means the improvement of the profitability of the enterprises assessed and the fulfillment of the institutional objectives.
\end{abstract}

\section{Palabras clave I keywords}

Estadístico, emprendimientos, análisis, estimación, métodos, prospectivos. Statistical, entrepreneurship, analysis, estimation, methods, prospective.

Cómo citar: Escobar Sailema, J.C. (2019). Modelo de estimación estadística «Programa Inclusión Productiva» Mipro-Ecuador. Retos Revista de Ciencias de la Administración y Economía, 9(18), 303-325. https://doi.org/ 10.17163/ret.n18.2019.08 


\section{Introducción y estado de la cuestión}

A escala mundial, la estimación estadística como parte de la ciencia económica es una de las áreas más importantes dentro del ámbito empresarial. Dichas estimaciones han permitido realizar interpretaciones de demanda del consumidor oportunas y confiables para la toma de decisiones (Cadena et al., 2017), así como también de disponer de información relevante sobre la sustitución de productos. Alvarado y Pinos (2017), Vergara et al., (2017) y García-Guerrero (2014) mencionan que el desarrollo de las estimaciones se analiza con variables sociodemográficas que inciden directamente sobre la demanda de un producto sea este un bien o servicio y consecuentemente algunas cuestiones de política económica que son la causa de las variaciones en el mercado de consumo, asumiendo de forma axiomática las preferencias de los consumidores hacia una demanda real como eje principal para los pronósticos en las empresas (Reyes-Sánchez, 2017).

Los métodos estadísticos en América Latina están siendo aplicados con más frecuencia y debatidos a gran escala en diversas áreas como la empresarial, agropecuaria, manufacturera, actividades productivas y de gobierno; siendo el eje fundamental en la toma de decisiones para los responsables de las empresas y/u organizaciones. Éstas se reflejan en el desarrollo económico-financiero de las mismas y el escenario futuro de la incidencia de sus factores. Al mismo tiempo, la aplicación errónea de métodos de estimación ha sido causa fundamental de las malas decisiones de niveles jerárquicos superiores, la mala planificación de la producción y por ende la baja rentabilidad. Los modelos más empleados han sido los de series de tiempo, implicando la utilización de una regresión lineal, promedios móviles simples, Suavización Exponencial Doble, Suavización Exponencial Simple, Método de Naïve, modelos ARIMA (Autoregressive Integrated Moving Average) entre otros. Estos modelos también son utilizados para la estimación sobre tendencias de rendimientos de diversos tipos de cultivos e incluso rendimientos de producción pecuaria (Delgadillo et al., 2016).

En el Ecuador, desde el 2013 se lleva a cabo el programa denominado «Inclusión Productiva» a cargo del Ministerio de Industrias y Productividad (MIPRO), en el cual se asesora a emprendimientos en los distintos sectores productivos con la elaboración de un plan de negocio y proyecciones de demanda; esto bajo un modelo de estimación financiera como componente intrínseco y sustancial del mismo, el cual se compone de una estructura financiera pronosticada a cinco años, tomando en consideración indicadores como la tasa de crecimiento de la producción, tasa de crecimiento del precio, tasa de descuento financiera, tasa de inflación, tasa de interés, plazo de financiamiento y otros que considera el especialista para su análisis y proyección. Con ello las estimaciones de los emprendimientos asesorados a escala nacional mantienen hasta hoy en día un error en la mayoría de los casos de más del veinte por ciento, dando a notar que el modelo y uso de herramientas de estimación de la demanda -pronóstico- utilizado, es cuanto menos, muy cuestionable, sobre todo en lo relativo al rendimiento real financiero de los emprendimientos implementados, dando como resultado un desempeño financiero desfavorable que conlleva a la baja rentabilidad. 
Valencia-Cruzaty (2017), Moscoso-Escobar y Botero-Botero (2013) y TocaTorres (2010), consideran al emprendimiento como la fórmula más empleada en tiempos de situación económica compleja, enmarcando a todos los sectores económicos y sociales para su desarrollo. Además, es reconocido como catalizador del crecimiento económico de los países, implementado tanto por la empresa privada internamente, como en el sector público (gobierno), creando nuevos productos, mercados, generando empleo y bienestar para la población.

Por consiguiente, se ve necesaria la implementación de nuevos modelos, metodologías, técnicas, herramientas e instrumentos de estimación para el desarrollo adecuado de los emprendimientos en términos de rentabilidad, además de asegurar una adecuada inversión y financiamiento, siendo importante al mismo tiempo obtener fuentes de información fiables, generando así efectividad en la predicción de eventos empresariales futuros. También es importante introducir al análisis factores de éxito que minimicen el riesgo en la implementación y funcionamiento del negocio como son: la producción en función a una demanda real, indicadores de estimación y métodos de pronósticos según el sector productivo.

El predecir el escenario futuro de las organizaciones y de todos los procesos productivos para un mejor desempeño financiero ha sido históricamente una labor netamente técnica y complicada, debido a que se requiere de una predicción óptima para un manejo adecuado de recursos y el aprovechamiento de factores productivos que inciden sobre la planificación. Con ello los empresarios han podido determinar la capacidad de la empresa para adquirir y producir la cantidad necesaria requerida, tanto de insumos como de productos demandados, y así predecir con anticipación el presupuesto necesario para evitar errores dentro de sus procesos, utilizando modelos basados en métodos cuantitativos contrastados con variables causales más el uso de probabilidades que se complementan para generar predicciones más certeras (LaoLeón et al., 2017).

Boada (2017a) presenta un estudio en el cual se comprueba a través de data real, cómo el Modelo Lineal Dinámico Bayesiano de Orden 1, puede ser aplicado sobre los residuos -diferencia entre las estimaciones y los históricos reales- tomados aleatoriamente de un Modelo de Regresión Múltiple, obteniendo de esa manera un complemento para el modelo de estimación estadística, el cual genera un factor que se retroalimenta de los residuos y se ajusta según la data histórica más reciente, unificando dos tendencias: la estadística bayesiana y la estadística frecuentista en un solo modelo, llegando a la conclusión que: a) la valoración o análisis más riguroso de variables causales como procedimiento de modelaje estadístico garantiza el poder de predicción de modelos de regresión múltiple, además; b) la simulación de escenarios futuros cercanos a la realidad, dependerá del uso correcto de las técnicas utilizadas en el modelo más las estrategias generadas por el predictor y, finalmente; c) se menciona que establecer un procedimiento de modelaje estadístico complementado con técnicas cuantitativas y cualitativas, sirven como herramienta fundamental para la creación de un modelo óptimo de predicción.

Moreno-Arenas (2016) plantea el diseño de un modelo de estimación estadística (pronóstico) a partir de métodos de series de tiempo. Dicho modelo pretende disminuir la variabilidad de la demanda de los insumos de empaque y embalaje, 
concluyendo que: a) el uso de métodos de serie de tiempo identifica con exactitud los cambios en la dinámica de la variable en función a obtener información fiable; b) la medición de las rotaciones de inventarios a partir de la estandarización de proyecciones es el resultado de un análisis de series temporales, lo que ayuda a mejorar rotundamente la situación de almacenamiento de la empresa y; c) los sistemas de planificación de recursos empresariales-ERP como fuente de información, mejoran el tipo de análisis y por ende disminuyen en gran medida la desviación de los datos.

Boada (2017b) expone el diseño de una herramienta automatizada de predicción en función de distintas variables de mercado. Ésta se sustenta en un análisis detallado de las variables causales identificadas, para posteriormente desarrollar un software denominado «Sistema de proyección de la demanda» para predecir las ventas, analizando precio, producto, facturación, fabricación, planificaciones futuras de gerencias estratégica de ventas, entre otras; concluyendo que el punto de partida de toda predicción es: a) el diseño y la adaptación de técnicas de estimación correctas, con el fin de obtener pronósticos más efectivos; b) considerando al sistema automatizado de proyección como una herramienta estadística y no como un competidor del estimador y; c) garantizando además la valoración, simulación y evaluación optima de diversos escenarios futuros, esto es la base fundamental de los planes a largo plazo de las distintas áreas de la empresa, como finanzas y contabilidad.

Sánchez y Gavira (2016) toman en consideración la jerarquía de la serie que contienen los componentes necesarios no observados de series de tiempos (variaciones aleatorias, ciclicidad, tendencia, y estacionalidad), esto para realizar una estimación de corto plazo, contribuyendo de esta manera al desarrollo de la teoría de pronóstico de series jerárquicas. Además, se determina el modelo más eficiente para pronosticar el ingreso monetario para el caso de estudio, utilizando como criterio el error promedio absoluto escalado (MASE), concluyendo que para el presente estudio: a) el método más eficiente de pronóstico es el ARIMA, sin descartar que varios métodos (TD-ETS, COMB-ARIMA, TD-ARIMA y MO-ARIMA) pueden ser empleados con un mejor resultado; b) la aplicación del método depende de la complejidad del estudio y la variabilidad de la serie; c) también evidenciando la importancia del contraste sobre la evaluación y determinación de los métodos más idóneos con otros enfoques.

Contreras et al. (2016) consideran a los pronósticos como una herramienta que proporciona una estimación cuantitativa de probabilidad, priorizando la elaboración de estimaciones futuras sobre el almacenamiento de productos perecederos debido al impacto económico-social que generan con estimaciones erróneas. De allí el interés en incorporar técnicas de pronósticos de series de tiempo con el objetivo de determinar el método más fiable, esto para estimar volúmenes de almacenamiento óptimos y poder prever los requerimientos en la cadena de suministros, concluyendo que: a) los pronósticos elaborados con la técnica de promedios móviles ponderados son los más aceptables en una planificación de movilidad de productos; b) garantizando así la disponibilidad de almacenamiento, la prolongación de caducidad y el aprovisionamiento de los mercados el mayor tiempo posible y; c) manteniendo un equilibrio entre demanda de mercado y producción.

Espino-Timón (2017) pretende determinar la existencia de herramientas open source que cumplan los requerimientos para un análisis predictivo y así la evaluación 
del funcionamiento de dichas herramientas en diferentes ambitos, las dos principales herramientas identificadas son: R, con la interface gráfica R-Studio, y Weka, que permiten detectar patrones en la data base para establecer previsiones futuras más óptimas. También se mencionan herramientas de análisis estadístico (SPSS, SAP Business Suite o SAS Software Package), usadas por grandes corporaciones, empresas, organizaciones, gobiernos y universidades, concluyendo que: a) $R$-Studio y Weka tiene mayor potencia y flexibilidad en cuanto a su aplicación, pudiendo ser utilizados para un análisis preliminar al modelo predictivo de la demanda, enfatizando b) el uso de los mismos para el manejo y procesamiento de bases de datos más grandes por su capacidad de almacenamiento y; c) se menciona una mejor gestión y análisis de RStudio como complemento a un análisis predictivo.

Arias-Vargas (2017) menciona al uso de la desviación estándar para el cálculo de un inventario de seguridad, en este caso se identifica al modelo de pronóstico como un promedio simple que tiene como efecto el incremento de la inversión en los inventarios de seguridad. Para evitar aquello, se propone la utilización de la desviación estándar de los errores del pronóstico asociado al modelo de pronóstico a aplicarse, concluyendo que: a) la importancia de la revisión de la desviación estándar de los errores en los modelos de pronósticos optimizan los inventarios; b) conjuntamente con el uso de softwares empresariales (ERP u otros) como complemento al modelo para el aseguramiento de los inventarios de la empresa; c) minimizando el impacto de la variabilidad de los datos en el inventario de seguridad, esto para alcanzar los niveles de servicio requeridos con inventarios de seguridad mínimos.

\section{Materiales y método}

Según Herrera et al. (2010), para la ejecución del presente trabajo de investigación se aplica como primer nivel la investigación descriptiva, que sirvió para examinar las características objeto de estudio, definir el tema y formular la hipótesis, además de seleccionar la técnica de recolección de data e identificar fuentes de consultas fiables, estableciendo en sí las causas y efectos del objeto de estudio.

Según Romero (2012), se aplica la investigación correlacional, donde se establece el grado de relación de las variables, modelo de estimación estadística (variable independiente) y rentabilidad (variable dependiente), estableciendo la incidencia que tiene la variable independiente en la solución del problema. Finalmente se emplea la investigación aplicada, que lleva el conocimiento teórico a lo práctico y a su vez a lo aplicativo, con la finalidad de mejorar las estimaciones de demanda de los emprendimientos asesorados dentro del programa «Inclusión Productiva» que lleva a cabo el Ministerio de Industrias y Productividad (MIPRO) del Ecuador.

\section{Población y muestra}

\subsection{Población}

La población está constituida por 702 emprendimientos a escala nacional, compuestos de 36 variables, conociéndose de esta manera con exactitud el número de elementos que comprende la población, determinando a la misma de carácter finita. 


\subsection{Muestra}

En la presente investigación, debido a la naturaleza del objeto de estudio, se aplica el tipo de muestreo probabilístico aleatorio estratificado con afijación proporcional, esto en cuanto se desea resaltar los subgrupos de emprendimientos en los distintos sectores productivos, observando las relaciones entre ellos y disminuyendo su variabilidad, manteniendo al mismo tiempo la proporcionalidad al tamaño de la población.

Tamaño de la muestra: $n=\frac{\sum_{i=1}^{1} N_{i} P_{i} Q_{i}}{N E+\frac{1}{N} \sum_{i=1}^{1} N_{i} P_{i} Q_{i}}$

Tamaño de cada estrato: $n_{i}=n\left[\frac{N_{i}}{\sum_{i=1}^{1} N_{i}}\right]=n\left(\frac{N_{i}}{N}\right)=n\left(W_{i}\right)$

Valor de estimación: $E=\frac{d^{2}}{Z_{1-\alpha / 2}{ }^{2}}$

Donde:

$$
\begin{aligned}
& \mathrm{N}=702 \\
& \mathrm{Z}=95 \% \text { de nivel de confianza equivalente a } 1,96 \text { valor } \mathrm{Z} \\
& \mathrm{E}=? \\
& \mathrm{n}=248 \\
& \mathrm{p}=0.5 \\
& \mathrm{q}=0.5 \\
& \mathrm{~d}^{\wedge} 2=0.05^{\wedge} 2 \\
& \mathrm{Z}_{1-\alpha / 2} 2=1.96^{\wedge} 2
\end{aligned}
$$

La muestra se calculó con un 95\% de nivel de confianza y una desviación estándar 0.5, para una población de 702 emprendimientos del programa de «Inclusión Productiva» que lleva a cabo el Ministerio de Industrias y Productividad (ver Tabla 1).

Tabla 1. Muestreo Estratificado con Afijación Proporcional

\begin{tabular}{|l|c|c|c|l|l|l|l|}
\hline \multicolumn{1}{|c|}{ Grupo } & Ni & \multicolumn{1}{c|}{$\mathbf{P i}$} & $\mathbf{Q i}$ & $\mathbf{P i} \mathbf{Q} \mathbf{i}$ & \multicolumn{1}{|c|}{$\mathbf{N} * \mathbf{P} * \mathbf{Q}$} & \multicolumn{1}{|c|}{ Wi } & \multicolumn{1}{c|}{$\mathbf{n i}$} \\
\hline Comercio bienes & 283 & 0.5 & 0.5 & 0.25 & 70.75 & 0.4031339 & 100 \\
\hline Comercio de servicios & 136 & 0.5 & 0.5 & 0.25 & 34 & 0.19373219 & 48 \\
\hline Manufactura & 182 & 0.5 & 0.5 & 0.25 & 45.50 & 0.25925926 & 64 \\
\hline Agropecuario & 101 & 0.5 & 0.5 & 0.25 & 25.25 & 0.14387464 & 36 \\
\hline Total & 702 & & & & 176 & 1 & 248 \\
\hline
\end{tabular}

Fuente: Elaboración propia a partir de data emprendimientos Setedis-Mipro

Según López (2014), para que una muestra sea representativa de su población debe contener al menos el 30\% de la totalidad de casos. En este sentido se aclara que, de 702 emprendimientos que comprende la población mediante la aplicación del 
método estadístico de muestreo estratificado, se obtienen 248 observaciones como cálculo muestral, totalmente representativo de la población con más del 30\% como establece el precitado autor (op. cit), por lo que queda evidenciado el cálculo de la muestra para el estudio presente.

\section{Análisis y resultados}

En el presente estudio se presenta información de 702 emprendimientos registrados en la Base [Data_Emprendimientos_Setedis_Mipro_xlsx] (data depurada), acumulativos desde el año 2013 al 2017, clasificados en 4 sectores productivos: Comercio de Bienes, Comercio de Servicios, Manufactura y Agropecuario (ver Tablas 2 y 3).

Tabla 2. Distribución por Provincia [Emprendimientos]

\begin{tabular}{|r|l|l|l|}
\hline No. & \multicolumn{1}{|c|}{ Provincias } & 18 & $\%$ \\
\hline $\mathbf{1}$ & Azuay & 31 & $2.6 \%$ \\
\hline $\mathbf{2}$ & Bolívar & 17 & $4.4 \%$ \\
\hline $\mathbf{3}$ & Cañar & 25 & $2.4 \%$ \\
\hline $\mathbf{4}$ & Carchi & 34 & $3.6 \%$ \\
\hline $\mathbf{5}$ & Chimborazo & 25 & $4.8 \%$ \\
\hline $\mathbf{6}$ & Cotopaxi & 36 & $3.6 \%$ \\
\hline $\mathbf{7}$ & El Oro & 27 & $5.1 \%$ \\
\hline $\mathbf{8}$ & Esmeraldas & 1 & $3.8 \%$ \\
\hline $\mathbf{9}$ & Galápagos & 42 & $0.1 \%$ \\
\hline $\mathbf{1 0}$ & Guayas & 28 & $6 \%$ \\
\hline $\mathbf{1 1}$ & Imbabura & 27 & $4 \%$ \\
\hline $\mathbf{1 2}$ & Loja & 33 & $3.8 \%$ \\
\hline $\mathbf{1 3}$ & Los Ríos & 103 & $4.7 \%$ \\
\hline $\mathbf{1 4}$ & Manabí & 13 & $14.7 \%$ \\
\hline $\mathbf{1 5}$ & Morona Santiago & 33 & $1.9 \%$ \\
\hline $\mathbf{1 6}$ & Napo & 21 & $4.7 \%$ \\
\hline $\mathbf{1 7}$ & Orellana & 12 & $3 \%$ \\
\hline $\mathbf{1 8}$ & Pastaza & 68 & $1.7 \%$ \\
\hline $\mathbf{1 9}$ & Pichincha & 23 & $9.7 \%$ \\
\hline $\mathbf{2 0}$ & Santa Elena & 27 & $3.3 \%$ \\
\hline $\mathbf{2 1}$ & Santo Domingo & 19 & $3.8 \%$ \\
\hline $\mathbf{2 2}$ & Sucumbíos & 23 & $2.7 \%$ \\
\hline $\mathbf{2 3}$ & Tungurahua & 16 & $3.3 \%$ \\
\hline $\mathbf{2 4}$ & Zamora Chinchipe & 702 & $2.3 \%$ \\
\hline & & $100 \%$ \\
\hline
\end{tabular}

Fuente: Elaboración propia a partir de data emprendimientos Setedis-Mipro

Según se observa en la Tabla 2, el mayor número de emprendimientos implementados se encuentran en la provincia de Manabí, con un 14.7\%, correspondiente a 
la implementación de 103 emprendimientos y un valor insignificante en la provincia de Galápagos con un $0.1 \%$. También se puede apreciar que las provincias donde más emprendimientos se implementan son las provincias de Pichincha (capital Quito) y Guayas (capital Guayaquil) con un 9.7\% y 6\% respectivamente, coincidentemente en que son las dos provincias con mayor densidad poblacional del Ecuador.

Tabla 3. Número de emprendimientos por Afijación Proporcional

\begin{tabular}{|c|c|c|c|}
\hline No. & Sectores Productivos & Frecuencia & $\%$ \\
\hline 1 & Comercio bienes & 100 & $40 \%$ \\
\hline 2 & Comercio de servicios & 48 & $19 \%$ \\
\hline 3 & Manufactura & 64 & $26 \%$ \\
\hline 4 & Agropecuario & 36 & $14 \%$ \\
\hline & Total & 248 & $100 \%$ \\
\hline
\end{tabular}

Fuente: Elaboración propia a partir de data emprendimientos Setedis-Mipro

En la Figura 1 se observa un porcentaje del 40.3\% de emprendimientos asesorados e implementados en el sector comercio de bienes, seguido del sector manufactura con el 25.9\%. Juntos representan más del 50\% de la variable en mención, mientras el sector servicios contempla el $19.4 \%$, y el sector agropecuario cubre el $14.4 \%$ a escala nacional. Esto evidencia que los emprendedores en su mayoría optan por emprender en negocios de naturaleza tangible-comercial.

Figura 1. Porcentaje de emprendimientos por Provincia

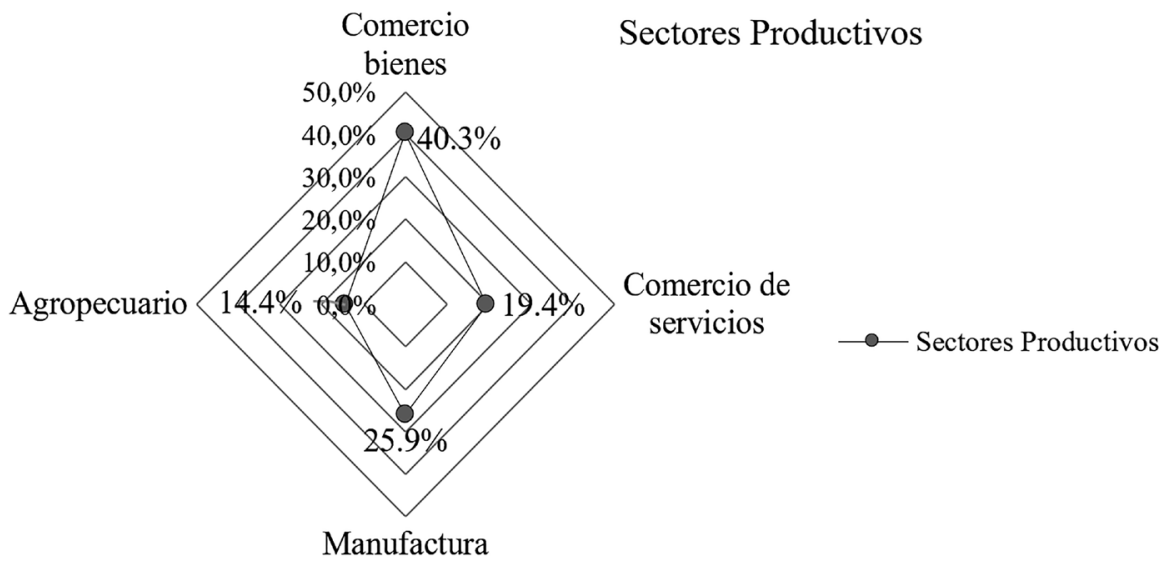

Fuente: Elaboración propia a partir de data emprendimientos Setedis-Mipro

A continuación se realiza el análisis sobre las variables; niveles error del pronóstico y rentabilidad de los emprendimientos (\% de utilidad o margen de utilidad y $\%$ de error como variable de incidencia). 
Tabla 4. Matriz General Errores sobre el Valor Real

\begin{tabular}{|l|l|l|l|l|l|l|}
\hline \multicolumn{1}{|c|}{ Factor } & \multicolumn{7}{c|}{ Sectores productivos } \\
\hline$e_{\mathrm{t}}$ & C.B & C.S & M & A & $\Sigma e_{\mathrm{t}}(+)(-)$ & $\%$ \\
\hline$e_{\mathrm{t}}(+)$ & 74 & 40 & 47 & 30 & 191 & $77 \%$ \\
\hline$e_{\mathrm{t}}(-)$ & 26 & 8 & 17 & 6 & 57 & $23 \%$ \\
\hline TOTAL & 100 & 48 & 64 & 36 & 248 & $100 \%$ \\
\hline
\end{tabular}

Fuente: Elaboración propia a partir de data emprendimientos Setedis-Mipro

- C.B: Comercio de bienes

- C.S: Comercio de Servicios

- M: Manufactura

- A: Agropecuarios

Según se evidencia en la Tabla 4, el porcentaje de subestimaciones en relación con el valor real llega al $77 \%$, dando a notar que las proyecciones realizadas en los emprendimientos asesorados fueron inferiores al valor real, mientras el porcentaje de sobrestimaciones llegó al 23\%, es decir, existieron emprendimientos que superaron el valor real alcanzado.

Tabla 5. Matriz general del porcentaje de error estadístico sobre las estimaciones

\begin{tabular}{|l|l|l|l|l|l|l|}
\hline \multicolumn{1}{|c|}{ Factor } & \multicolumn{7}{c|}{ Sectores productivos } \\
\hline$e_{\mathrm{t}}$ & C.B & C.S & $\mathrm{M}$ & $\mathrm{A}$ & $\Sigma e_{\mathrm{t}}<;>10 \%$ & $\%$ \\
\hline$e_{\mathrm{t}}<10 \%$ & 5 & 2 & 3 & 2 & 12 & $5 \%$ \\
\hline$e_{\mathrm{t}}>10 \%$ & 95 & 46 & 61 & 34 & 236 & $95 \%$ \\
\hline TOTAL & 100 & 48 & 64 & 36 & 248 & $100 \%$ \\
\hline
\end{tabular}

Fuente: Elaboración propia a partir de data emprendimientos Setedis-Mipro

Según se observa en la Tabla 5, el 95\% de los emprendimientos sobre 248 observaciones sobrepasan el $10 \%$ de margen de error en sus predicciones. Tan solo el 5\% de los pronósticos se encuentran dentro del margen de error estadísticamente aceptable. Según Lamas (2016), el error estadístico para cualquier estudio de estimación se encuentra entre 0-10\% del margen de error máximo, esto en función al nivel de confianza apropiado; sin embargo, dependerá del investigador definir el margen de error admitido, tomando a consideración el tipo de estimación y los factores que incidan en las mismos.

\subsection{Análisis del error de pronóstico por sectores productivos}

Se procede a realizar el análisis respectivo sobre $\boldsymbol{e} \%=\left(\frac{\boldsymbol{y}_{\boldsymbol{t}}-\widehat{\boldsymbol{y}}_{\boldsymbol{t}}}{\boldsymbol{y}_{\boldsymbol{t}}}\right) * \mathbf{1 0 0} \quad$ (Hanke, 2015).
Donde:

$\boldsymbol{e}_{\boldsymbol{t}}$ : error del pronóstico

$\boldsymbol{Y}_{t}$ : valor real

$\widehat{\boldsymbol{Y}}_{\boldsymbol{t}}:$ valor del pronóstico 
Tabla 6. Análisis Descriptivo Error (\%) Sector Comercio de Bienes

\begin{tabular}{|l|l|}
\hline \multicolumn{1}{|c|}{ Estadísticos } & \multicolumn{1}{c|}{ Valores } \\
\hline N.-Validos & 100 \\
\hline Perdidos & 0 \\
\hline Media & 61.72 \\
\hline Error estándar media & 2.831 \\
\hline Mediana & 68 \\
\hline Moda & 97 \\
\hline Desviación estándar & 28.312 \\
\hline Varianza & 801.577 \\
\hline Asimetría & -.552 \\
\hline Error estándar de asimetría & .241 \\
\hline Curtosis & -.885 \\
\hline Error estándar de curtosis & .478 \\
\hline Rango & 94 \\
\hline Mínimo & 3 \\
\hline Máximo & 97 \\
\hline Percentiles .25 & 37.25 \\
\hline .50 & 68.00 \\
\hline .75 & 84.75 \\
\hline
\end{tabular}

Fuente: Elaboración propia a partir de data emprendimientos Setedis-Mipro

Según los estadísticos descriptivos que se presentan en la Tabla 6, el promedio de error sobre 100 observaciones es de $61.72 \%$, con una desviación estándar de $28.312 \%$. Además, se puede apreciar que la mitad de los emprendimientos tuvieron un porcentaje de error en sus pronósticos menores al $68 \%$, mientras que $97 \%$ fue el porcentaje de error más frecuente en una escala de 1-100 por ciento, la diferencia entre el porcentaje mínimo y máximo de error del pronóstico fue $94 \%$, y el 50 por ciento de los emprendimientos tuvieron un error en sus pronósticos entre $37.25 \%$ y $88.75 \%$. La distribución es asimétrica negativa, es decir la mayor cantidad de datos se encuentran agrupados a la derecha y tiene una curtosis platicúrtica, lo que significa que existe muy poca agrupación de datos en la región central, pudiendo identificar que los datos presentan gran variabilidad o dispersión en relación a su media.

Tabla 7. Análisis Descriptivo Error (\%) Sector Comercio de Servicios

\begin{tabular}{|l|l|}
\hline \multicolumn{1}{|c|}{ Estadísticos } & \multicolumn{1}{c|}{ Valores } \\
\hline N.-Validos & 48 \\
\hline Perdidos & 0 \\
\hline Media & 64.13 \\
\hline Error estándar media & 3.293 \\
\hline Mediana & 72.50 \\
\hline
\end{tabular}




\begin{tabular}{|l|l|}
\hline \multicolumn{1}{|c|}{ Estadísticos } & \multicolumn{1}{c|}{ Valores } \\
\hline Moda & 79.00 \\
\hline Desviación estándar & 22.813 \\
\hline Varianza & 520.452 \\
\hline Asimetría & -1.004 \\
\hline Error estándar de asimetría & .343 \\
\hline Curtosis & .704 \\
\hline Error estándar de curtosis & .674 \\
\hline Rango & 96 \\
\hline Mínimo & 0.3 \\
\hline Máximo & 96 \\
\hline Percentiles .25 & 48.50 \\
\hline .50 & 72.50 \\
\hline .75 & 79.00 \\
\hline
\end{tabular}

Fuente: Elaboración propia a partir de data emprendimientos Setedis-Mipro

Según se puede observar en la Tabla 7, el promedio de error sobre 48 observaciones es de 64.13 por ciento, con una desviación estándar de $22.813 \%$. Además, se puede apreciar que la mitad de los emprendimientos tuvieron un porcentaje de error en sus pronósticos menores al 72.50\%. 79 fue el porcentaje de error más frecuente en una escala de 1-100 por ciento; mientras que la diferencia entre el porcentaje mínimo y máximo de error del pronóstico fue 96 por ciento, y el 50\% de los emprendimientos tuvieron un error en sus pronósticos entre $48.50 \%$ y 79\%. La distribución es asimétrica negativa, es decir la mayor cantidad de datos se encuentran agrupados a la derecha y tiene una curtosis leptocúrtica, por consiguiente, existen mayor cantidad de datos en la región central, identificando con ello que los datos rodean en gran medida el $64.13 \%$ de error del pronóstico.

Tabla 8. Análisis Descriptivo Error (\%) Sector Agropecuario

\begin{tabular}{|l|l|}
\hline \multicolumn{1}{|c|}{ Estadísticos } & \multicolumn{1}{c|}{ Valores } \\
\hline N.-Validos & 36 \\
\hline Perdidos & 0 \\
\hline Media & 57.94 \\
\hline Error estándar media & 4.482 \\
\hline Mediana & 69 \\
\hline Moda & 83 \\
\hline Desviación estándar & 26.891 \\
\hline Varianza & 723.140 \\
\hline Asimetría & -.512 \\
\hline Error estándar de asimetría & .393 \\
\hline Curtosis & -1.134 \\
\hline Error estándar de curtosis & .768 \\
\hline
\end{tabular}




\begin{tabular}{|l|l|}
\hline \multicolumn{1}{|c|}{ Estadísticos } & \multicolumn{1}{c|}{ Valores } \\
\hline Rango & 87 \\
\hline Mínimo & 5 \\
\hline Máximo & 92 \\
\hline Percentiles .25 & 36 \\
\hline .50 & 69 \\
\hline .75 & 81 \\
\hline
\end{tabular}

Fuente: Elaboración propia a partir de data emprendimientos Setedis-Mipro

Según los datos del análisis descriptivo de la Tabla 8, el promedio de error sobre 36 observaciones es de 57.94\% con una desviación estándar de 26.89. Además, se puede apreciar que la mitad de los emprendimientos tuvieron un porcentaje de error en sus pronósticos menores al 69\%.83 fue el porcentaje de error más frecuente en una escala de 1-100 por ciento, la diferencia entre el porcentaje mínimo y máximo de error del pronóstico fue 87 por ciento, y el 50\% de los emprendimientos tuvieron un error en sus pronósticos entre 36\% y 81\%. La distribución es asimétrica negativa, es decir la mayor cantidad de datos se encuentran agrupados a la derecha y tiene una curtosis platicúrtica, por consiguiente, se puede evidenciar que existe muy poca agrupación de datos alrededor del 57.94\% de error del pronóstico.

Tabla 9. Análisis Descriptivo Error (\%) Sector Manufactura

\begin{tabular}{|l|l|}
\hline \multicolumn{1}{|c|}{ Estadísticos } & \multicolumn{1}{c|}{ Valores } \\
\hline N.-Validos & 64 \\
\hline Perdidos & 0 \\
\hline Media & 71.19 \\
\hline Error estándar media & 3.320 \\
\hline Mediana & 78.00 \\
\hline Moda & 97 \\
\hline Desviación estándar & 26.556 \\
\hline Varianza & 705.234 \\
\hline Asimetría & -.903 \\
\hline Error estándar de asimetría & .299 \\
\hline Curtosis & -.099 \\
\hline Error estándar de curtosis & .590 \\
\hline Rango & 94 \\
\hline Mínimo & 4 \\
\hline Máximo & 98 \\
\hline Percentiles .25 & 51.50 \\
\hline .50 & 78 \\
\hline .75 & 97 \\
\hline
\end{tabular}

Fuente: Elaboración propia a partir de data emprendimientos Setedis-Mipro 
Según los estadígrafos de la Tabla 9, el promedio de error sobre 64 observaciones es de 71.19 por ciento, con una desviación estándar de $26.55 \%$. Además, se puede apreciar que la mitad de los emprendimientos tuvieron un porcentaje de error en sus pronósticos menores al 78\%. 97 fue el porcentaje de error más frecuente en una escala de 1-100 por ciento. La diferencia entre el porcentaje mínimo y máximo de error del pronóstico fue 94 por ciento, y el 50\% de los emprendimientos tuvieron un error en sus pronósticos entre 51.50\% y 97\%. La distribución es asimétrica negativa, es decir la mayor cantidad de datos se encuentran agrupados a la derecha y tiene una curtosis platicúrtica, en consecuencia existe muy poca agrupación de datos o mucha variabilidad alrededor de la media $71.19 \%$.

\subsection{Análisis de rentabilidad por estratos}

Para el análisis de rentabilidad se toma a consideración el \% U o margen de utilidad como variable compuesta $\% \mathbf{U}=\frac{\mathbf{U N}}{\mathbf{V T}}$ la cual es procesada y representada en el siguiente análisis. Según Daza- Izquierdo (2016), la rentabilidad es una condición, capacidad o aptitud de las organizaciones para generar u obtener renta partiendo de una inversión. Es decir, es la culminación de un resultado de cualquier actividad económica, considerando que la rentabilidad es la interpretación de los beneficios, ganancias, utilidad, y excedente en términos porcentuales o absolutos en función a determinados indicadores. En este caso la condición de rentabilidad se establece en función a la utilidad porcentual o margen de utilidad.

Tabla 10. Representación Numérica y Porcentual de Utilidad

\begin{tabular}{|l|l|l|l|l|l|l|}
\hline \multicolumn{1}{|c|}{ Factor } & \multicolumn{7}{c|}{ Sectores Productivos } \\
\hline Utilidad & C.B & C.S & M & A & $\Sigma$ Utilidad & $\%$ \\
\hline U (-) & 22 & 8 & 23 & 14 & 67 & $27 \%$ \\
\hline U (+) & 78 & 40 & 41 & 22 & 181 & $73 \%$ \\
\hline Total & 100 & 48 & 64 & 36 & 248 & $100 \%$ \\
\hline
\end{tabular}

Fuente: Elaboración propia a partir de data emprendimientos Setedis-Mipro

$\mathbf{U N}=$ Utilidad neta

VT $=$ Ventas totales

Unidades $=$ Porcentual

Según se evidencia en la Tabla 10, el 73\% de los emprendimientos asesorados obtienen utilidad, mientras el $27 \%$ generan pérdida. Además, se puede observar con meridiana claridad que los sectores donde se concentra la mayor cantidad de emprendimientos son el de Comercio de Bienes y Manufactura.

Según López (2016), las distintas formas de «hacer empresa» hacen que los márgenes de utilidad tengan gran diversidad y disparidad entre las unidades de negocio e incluso entre sectores productivos. El autor (op. cit.) menciona que un aceptable o buen margen de utilidad es el que supera el $20 \%$, indicando además que comúnmente las industrias con empresas competitivas obtienen márgenes pequeños de utilidad, por lo que para ser rentables generan grandes volúmenes de ventas. En cambio, en pequeños negocios innovadores se puede generalmente obtener márge- 
nes de utilidad mayores, esto debido a que los volúmenes de venta aumentan por el número reducido de competidores. También se considera el aumento de margen de utilidad por la aplicación de economías de escala.

Tabla 11. Medición del Î́ndice de Rentabilidad Modelo Actual

\begin{tabular}{|l|l|l|l|l|}
\multicolumn{1}{c|}{$\begin{array}{c}\text { Sectores } \\
\text { productivos }\end{array}$} & \multicolumn{1}{|c|}{ Utilidad $<\mathbf{2 0 \%}$} & \multicolumn{1}{|c|}{ Utilidad $\mathbf{2 0} \%$} & $\begin{array}{c}\text { Utilidad (-) } \\
\text { perdida }\end{array}$ & Total \\
\hline C.B & 21 & 57 & 22 & 100 \\
\hline C.S & 4 & 36 & 8 & 48 \\
\hline M. & 19 & 22 & 23 & 64 \\
\hline A. & 4 & 18 & 14 & 36 \\
\hline Total Numérico & 48 & 133 & 67 & 248 \\
\hline Total Porcentual & $19 \%$ & $54 \%$ & $27 \%$ & $100 \%$ \\
\hline
\end{tabular}

Fuente: Elaboración propia a partir de data emprendimientos Setedis-Mipro

Según se evidencia en la Tabla 11, el 54\% de los emprendimientos asesorados obtienen una utilidad mayor al 20\% considerada aceptable, mientras que el 27\% generan pérdida, y el $19 \%$ generan una utilidad menor al $20 \%$, considerado como poco apreciable.

\subsection{Evaluación del modelo actual vs. el modelo propuesto}

Según Render et al., (2015), para realizar la evaluación de un modelo de estimación empleado, se consideran los siguientes elementos que son constratados con los del modelo actual.

Tabla 12. Ponderación Elementos del Modelo de Estimación «Método de Holmes»

\begin{tabular}{|c|l|l|l|l|l|l|l|l|l|}
\hline Comp. & $\mathbf{C 1}$ & $\mathbf{C 2}$ & $\mathbf{C 3}$ & $\mathbf{C 4}$ & $\mathbf{C 5}$ & $\mathbf{C 6}$ & Total & Orden & Proporción \\
\hline $\mathbf{C 1}$ & - & 1 & 1 & 1 & 1 & 1 & 5 & 1 & $33.33 \%$ \\
\hline C2 & 0 & - & 0.50 & 0.75 & 0.75 & 0.75 & 2.75 & 2 & $18.33 \%$ \\
\hline C3 & 0 & 0.50 & - & 0.50 & 0.50 & 0.50 & 2 & 3 & $13.33 \%$ \\
\hline C4 & 0 & 0.25 & 0.50 & - & 0.75 & 0.50 & 2 & 3 & $13.33 \%$ \\
\hline C5 & 0 & 0.25 & 0.50 & 0.25 & - & 0.75 & 1.75 & 4 & $11.67 \%$ \\
\hline C6 & 0 & 0.25 & 0.50 & 0.50 & 0.25 & - & 1.50 & 2 & $10 \%$ \\
\hline
\end{tabular}

Fuente: Elaboración propia a partir de data emprendimientos Setedis-Mipro

1: Muy importante

0.75: Significativamente importante

0,50: Importante

0,25: Significativamente menos importante

0 : Poco importante
C1: Obtención de datos de entrada

C2: Desarrollo de una solución

C3: Prueba de solución

C4: Análisis de resultados

C5: Análisis de sensibilidad 
Tabla 13. Evaluación Modelo Actual

\begin{tabular}{|l|l|l|l|l|} 
No. & \multicolumn{1}{|c|}{ Componentes } & \multicolumn{1}{|c|}{ Descripción } & Orden & \multicolumn{1}{|c|}{ Valoración } \\
\hline 1 & Obtención de datos de entrada & $\begin{array}{l}\text { Procedimientos } \\
\text { estadísticos }\end{array}$ & 1 & - \\
\hline 2 & Desarrollo de una solución & Manipulación del modelo & 2 & $18 \%$ \\
\hline 3 & Prueba de solución & Pruebas estadísticas & 3 & - \\
\hline 4 & Análisis de resultados & $\begin{array}{l}\text { Determinación de las im- } \\
\text { plicaciones de la solución }\end{array}$ & 3 & $13 \%$ \\
\hline 5 & Análisis de sensibilidad & Pruebas de Sensibilidad & 4 & $12 \%$ \\
\hline 6 & Implementación de resultados & $\begin{array}{l}\text { Incorporación de la } \\
\text { solución }\end{array}$ & 2 & $10 \%$ \\
\hline & & $-12 \%$ \\
\hline
\end{tabular}

Fuente: Elaboración propia a partir de data emprendimientos Setedis-Mipro

Como se observa en la Tabla 13, la efectividad del modelo actual es del 53\%, considerando para el componente $\mathrm{C} 1$ una probabilidad de 0.5 de fracaso y 0.5 de éxito en el procedimiento. Esto por suponerse que existan problemas de manipulación e ingreso de data errónea, sin embargo, para el MEED-JCES-2018, se establece un procedimiento para la gestión de riesgos que mitigue este tipo de eventualidades.

\subsection{Metodología del modelo operativo propuesto «MEED-JCES-2018»}

Según Render et al., (2015, pp.31-34) los modelos "deben ser totalmente funcionales, faciles de resolver y comprender, ser realistas y modificables, además de ser factibles en la obtención de requerimiento de datos de entrada". Enfatizan además los autores que el desarrollo del modelo debe ser minucioso para que se pueda resolver y se asemeje más a la realidad de la predicción.

El modelo propuesto se denominará en lo sucesivo «MEED-JCES-2018». Dicho modelo abarcará los enfoques y aportaciones de diferentes autores, y se evidenciará con mayor énfasis métodos cuantitativos de pronósticos, específicamente los de series de tiempo. Adicional de aquello, se presentan parámetros financieros y estadísticos que permitirán medir con precisión la exactitud de los pronósticos y el nivel de rentabilidad (ver Figura 2). 


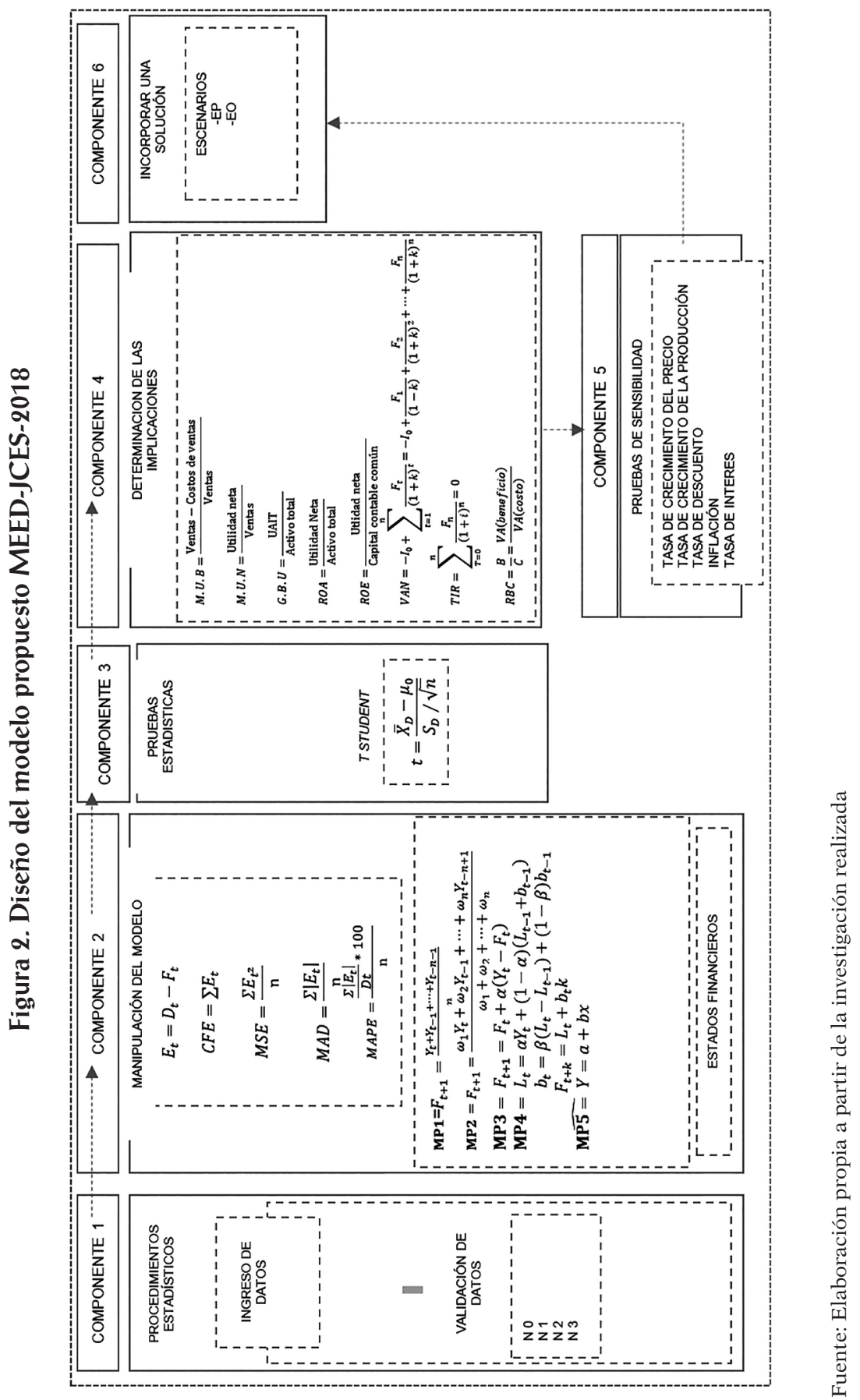

ISSN impreso: 1390-6291; ISSN electrónico: 1390-8618 
1. Ingreso de input-data: para el ingreso de datos se establece la metodología propuesta en función a niveles de validación, posterior a dicha validación la data ingresa al «MEED-JCES-2018».

2. Manipulación del modelo: se procesa la data, se establece el error que más se ajusta a dicha serie y se aplica el método que menos error presente (para la identificación del menor error se toma a consideración el MAPE por la facilidad de interpretación).

3. Prueba estadística: se obtienen los datos estadísticos arrojados por el «MEEDJCES-2018» sobre la aplicación de los distintos métodos de pronósticos, para ser comparados con los datos que arroje el software IBM SPSS o Minitab (en este caso se pone a consideración el uso de otro tipo de software propuesto por el analista). La finalidad de esta comparación es para identificar qué tan efectivo es el modelo.

4. Determinación de las implicaciones: se realiza un análisis de los indicadores de rentabilidad y se determina que tan rentable es el negocio en función a los datos obtenidos.

5. Prueba de sensibilidad: la prueba de sensibilidad comprende la manipulación de las tasas de crecimiento del precio, producción, inflación, tasa de descuento.

6. Incorporar la solución: en la incorporación de la solución se recomienda establecer al menos 2 escenarios (pesimistas- optimistas), a partir de la sensibilidad de las variables.

Finalmente, se sugiere para la fase de predicción estadística, contrastar los resultados con las herramientas SPSS, R-Studio, Minitab, y, Stat-Graphics.

\subsection{Contraste del modelo actual vs el modelo propuesto}

A continuación, como interpretación de los resultados finales, se presenta el análisis sobre el contraste de los elementos antes y después de la aplicación del «MEED-JCES-2018».

Tabla 14. Contraste de la Aplicación del Modelo \% U

\begin{tabular}{|l|l|l|}
\hline \multicolumn{1}{|c|}{ Estadísticos } & \multicolumn{1}{|c|}{$\begin{array}{c}\text { Antes de la aplicación } \\
\text { del MEED }\end{array}$} & $\begin{array}{c}\text { Después de la } \\
\text { aplicación del MEED }\end{array}$ \\
\hline N.-Validos & 248 & 248 \\
\hline Perdidos & 0 & 0 \\
\hline Media & 32.1008 & 46.8226 \\
\hline Mediana & 28.8000 & 44.0000 \\
\hline Moda & 64.00 & 74.00 \\
\hline Desviación estándar & 21.31471 & 19.26968 \\
\hline Varianza & 454.317 & 371.321 \\
\hline Asimetría & .501 & .878 \\
\hline Error estándar de asimetría & .155 & .155 \\
\hline Curtosis & -.499 & .608 \\
\hline
\end{tabular}




\begin{tabular}{|l|l|l|}
\hline \multicolumn{1}{|c|}{ Estadísticos } & \multicolumn{1}{|c|}{$\begin{array}{c}\text { Antes de la aplicación } \\
\text { del MEED }\end{array}$} & $\begin{array}{c}\text { Después de la } \\
\text { aplicación del MEED }\end{array}$ \\
\hline Error estándar de curtosis & .308 & .308 \\
\hline Rango & 97.00 & 97.00 \\
\hline Mínimo & .00 & 20.00 \\
\hline Máximo & 97.00 & 117.00 \\
\hline Percentiles .25 & 16.0000 & 31.0000 \\
\hline .75 & 48.9500 & 59.0000 \\
\hline
\end{tabular}

Fuente: Elaboración propia a partir de data emprendimientos Setedis-Mipro

\subsection{Verificación de hipótesis}

Paso 1.-Planteamiento de Hipótesis

Ho: La variable $e_{t}^{\%}$ (error relativo) no incide significativamente en la rentabilidad ( $\%$ U de o margen de utilidad) de los emprendimientos $\left(\tilde{x}_{1}=\tilde{x}_{2}\right)$

H1: La variable $e_{t}^{\%}$ (error relativo) sí incide significativamente en la rentabilidad (\% U o margen de utilidad) de los emprendimientos $\left(\tilde{x}_{1} \neq \tilde{x}_{2}\right)$

\section{Paso 2.-Niveles de Significación}

Nivel de confianza: $95 \%, \alpha=100 \%-95 \%=5 \%=0.05$.

Error tipo 1. - Probabilidad de rechazo de Ho cuando es verdadera.

Error tipo 2. - Probabilidad de aceptar de Ho cuando es falsa

Paso 3.-Estadístico de Prueba

Si $\mathrm{p}<=0.05$ se rechaza Ho

Si $\mathrm{p}>=0.05$ se acepta Ho

Paso 4.-Regla de Decisión

Si $\mathrm{p}<=0.05$ se rechaza Ho y se acepta la H1.

Se procede a realizar el ritual de significancia estadística para la selección de la prueba de hipótesis. Se aplica la prueba de Kolmogórov-Smirnov, para una muestra sobre la variable diferencia ( $e_{t} \%$ de error del pronóstico antes- $e_{t} \%$ después de la aplicación del MEED) y sobre la variable diferencia (\% U modelo actual - \% U modelo propuesto), con el fin de comprobar si las variables de estudio se distribuyen o pertenecen a una distribución normal. 
Tabla 15. Prueba de Kolmogórov-Smirnov para una Muestra

\begin{tabular}{|l|l|l|l|}
\hline \multicolumn{1}{|c|}{ Factores } & \multicolumn{1}{|c|}{ Descriptivos } & Diferencia $\boldsymbol{~}_{t} \%$ & Diferencia $\% \mathbf{U}$ \\
\hline $\mathrm{N}$ & & 248 & 248 \\
\hline Parámetros normales ${ }^{\mathrm{a}, \mathrm{b}}$ & Media & 37.8560 & -14.7218 \\
\hline & Desv. & 21.62097 & 3.52447 \\
\hline Máximas diferencia & Absoluta & .202 & .219 \\
\hline Extremas & Positiva & .153 & .219 \\
\hline & Negativa & -.202 & .206 \\
\hline Estadístico de prueba & & .202 & .219 \\
\hline Sig. asintónica (bilateral) & & $.000^{\mathrm{c}}$ & $.000^{\mathrm{c}}$ \\
\hline
\end{tabular}

Fuente: Elaboración propia a partir de data emprendimientos Setedis-Mipro

a. La distribución de prueba es normal.

b. Se calcula a partir de datos.

c. Corrección de significación de Lilliefors.

Ho: Hipótesis de homogeneidad

H1: de diferencias

En la Tabla 15 se puede evidenciar un valor p de $0.00<0.05$, lo que nos permite rechazar la Ho y aceptar la H1, y se confirma que no existe homogeneidad, es decir, la distribución de los datos de las variables en estudio no es iguales en su estructura ni en su forma -no pertenecen a una distribución normal-, por lo que se aplicará la prueba de rangos de Wilcoxon.

A continuación, en la Tabla 16, se presenta la hipótesis sujeta a verificación con la prueba de rangos de Wilcoxon para muestras relacionadas. Ésta se utilizó para comparar dos mediciones de rangos (medianas), con el fin de determinar que la diferencia no se deba al azar.

Tabla 16. Prueba de Rangos de Wilcoxon

\begin{tabular}{|c|c|c|c|c|}
\hline Descriptivos & Diferencia & $\mathbf{N}$ & $\begin{array}{c}\text { Rango } \\
\text { Promedio }\end{array}$ & $\begin{array}{l}\text { Suma de } \\
\text { Rangos }\end{array}$ \\
\hline $\begin{array}{l}\% \text { Error modelo propuesto- } \\
\% \text { Error modelo actual }\end{array}$ & $\begin{array}{l}\text { Rangos negativos } \\
\text { Rangos positivos } \\
\text { Empates } \\
\text { Total }\end{array}$ & $\begin{array}{l}237^{\mathrm{a}} \\
11 \mathrm{~b} \\
0 \mathrm{c} \\
248\end{array}$ & $\begin{array}{l}128.39 \\
40.64\end{array}$ & $\begin{array}{l}30429.00 \\
447.00\end{array}$ \\
\hline $\begin{array}{l}\% \text { de Utilidad del modelo propuesto- } \\
\% \text { Utilidad del modelo actual }\end{array}$ & $\begin{array}{l}\text { Rangos negativos } \\
\text { Rangos positivos } \\
\text { Empates } \\
\text { Total }\end{array}$ & $\begin{array}{l}0 \mathrm{a} \\
248 \mathrm{~b} \\
0 \mathrm{c} \\
248\end{array}$ & $\begin{array}{l}0.00 \\
124.50\end{array}$ & $\begin{array}{l}00.00 \\
30876.00\end{array}$ \\
\hline
\end{tabular}

Fuente: Elaboración propia a partir de data emprendimientos Setedis-Mipro 
Tabla 17. Estadísticos de Prueba

\begin{tabular}{|l|l|l|}
\hline \multicolumn{1}{|c|}{ Descripción } & \multicolumn{1}{|c|}{$\mathbf{Z}$} & \multicolumn{1}{c|}{ Sig. asintótica (bilateral) } \\
\hline $\begin{array}{l}\text { \% Error con aplicabilidad del MEED } \\
\text { \% Error antes de la aplicabilidad del } \\
\text { MEED }\end{array}$ & $-13.377^{\mathrm{b}}$ & .000 \\
\hline $\begin{array}{l}\text { \% de Utilidad del modelo propuesto- } \\
\% \text { Utilidad del modelo actual }\end{array}$ & '13.795 & .000 \\
\hline
\end{tabular}

Fuente: Elaboración propia a partir de data emprendimientos Setedis-Mipro

a. Prueba de Wilcoxon de los rangos con signo

b. Se basa en rangos positivos

\section{Paso 5.-Toma de Decisión}

Como se puede observar en la Tabla 17, el sig. es $0.00<0.05$, lo que evidencia la existencia de diferencias entre las dos evaluaciones sobre el mismo grupo de emprendimientos, siendo el valor $\mathrm{Z}$ mayor que el nivel de la confianza al 95\%=1.96. Es decir, el valor $\mathrm{Z}$ calculado de $-13.377^{\mathrm{b}}$ y $13.795^{\mathrm{b}}$ cae en la zona de rechazo a dos colas de distribución, por lo que se puede corroborar que existe un cambio significativo en el \% de error del pronóstico y del porcentaje de utilidad luego de la aplicación del MEED, por lo tanto, rechazamos Ho y aceptamos H1.

\section{Discusión y conclusiones}

Los resultados obtenidos en los pronósticos con el modelo propuesto nos dan cada vez predicciones más precisas. Éstas provienen de un modelo desagregado que se basa en las medidas sobre el error cuadrático medio-ECM y error absoluto medioPEAM, dependiendo en gran medida del equilibrio entre el grado de incertidumbre y heterogeneidad de sus datos (López et al., 2017).

Según Render et al. (2015, pp.31-34) "los modelos deben ser totalmente funcionales, fáciles de resolver y comprender, ser realistas y modificables, además de ser factibles en la obtención de requerimiento de datos de entrada". Enfatizan los autores (op. cit.) además que el desarrollo del modelo debe ser minucioso para que se lo pueda resolver y se asemeje más a la realidad de predicción. Una vez examinados los trabajos y publicaciones de varios autores, se desarrolló el modelo de estimación estadística de la demanda denominado "MEED-JCES-2018», que establece factores primordiales para una predicción óptima y una estructura para un manejo práctico, empezando con un sistema de información que analiza la tendencia de la serie a pronosticar, la aplicación automática de métodos de series de tiempo como: El método de promedio móvil simple, promedio móvil ponderado, suavizamiento exponencial, suavización exponencial doble y regresión lineal, que conjuntamente con indicadores estadísticos financieros y de control, ayudan al mejoramiento de las estimaciones para detectar la presencia de errores, tomar acciones correctivas y tomar mejores decisiones. 
La propuesta de un modelo para el cálculo de las estimaciones contribuye a las empresas en la disminución del abastecimiento excesivo, la minimización de los costos de materiales, producción y productos terminados. La aplicación de un modelo implica la evaluación y comparación de distintos métodos cuantitativos, con el objetivo de obtener mayor precisión en cuanto a su estimación, siendo el método más empleado el Método Winter (Garduño-García, 2011).

En los modelos investigados se explica claramente su estructura. En el caso particular el modelo denominado SysPPAc compuesto por: un sistema de información, análisis tendencial, métodos de estimación estadística, indicadores de selección de métodos y generación de información; este modelo es netamente funcional ya que migra y clasifica data histórica, aplica modelos matemáticos de estimación basados en series de tiempo, promedios-móviles y suavización-exponencial, selecciona un número de períodos anteriores, el factor de ajuste para la valoración de cualquier patrón y/o tendencia de la serie a ser estimada, valida el modelo, genera información fundamental fiable y de manera automática (Montañez-Muñoz, 2010).

Tomando en consideración el componente de tendencia, los errores de las estimaciones y la autocorrelación histórica, lo aconsejable es trabajar en función de una serie totalmente diferenciada y reducida en su varianza a través de la aplicación del logaritmo natural -serie retrasada en un periodo-, esto se ve reflejado en la mayoría de estudios de pronósticos y modelos de estimaciones (Peña Figueroa \& Paredes Mora, 2016).

Se establece la utilización de herramientas estadísticas y de probabilidad para el análisis del comportamiento de la $\bar{x}, S$, el rango, el percentil 90 y el percentil 10 -los dos definen la probabilidad de ocurrencia del $80 \%$-, en base a este análisis y la utilización de métodos de pronósticos se construyen series de tiempo y se establecen parámetros de desempeño, se define a través del análisis de errores el método que modela de mejor manera el comportamiento de la serie. Finalmente, se evidencia que los valores pronosticados difieren de manera insignificante a los reales, es decir, existe una buena aproximación para predecir valores futuros, concluyendo que el análisis minucioso de estadígrafos aporta en gran medida a la definición o selección del modelo más adecuado y consecuentemente la aproximación más óptima al valor real (Silva-Romero, 2013).

Se recomienda la actualización del modelo con la inclusión de nuevos métodos, técnicas y herramientas estadísticas para el muestreo, procesamiento, análisis y recolección de datos, así también como para el contraste de hipótesis. De la misma manera se recomienda el uso de técnicas de control y seguimiento, la inclusión de indicadores estadísticos y financieros que garanticen la calidad de la información generada por el modelo. Además, se sugiere mantener una data-base de buena calidad que sirva para posteriores análisis, entendiéndose por buena calidad de los datos: su disponibilidad, usabilidad, confiabilidad, pertinencia y calidad en su presentación. Asimismo, realizar una evaluación periódica del "MEED-JCES-2018», con el objeto de mejorar el mismo a través de la inserción de nuevas herramientas o aplicaciones informáticas (software estadístico) para el proceso de predicción, dichas mejoras deben sustentarse lógica, técnica y científicamente. 


\section{Referencias}

Alvarado, J. L., \& Pinos, O. A. (2017). Estimación de ingresos de la población ecuatoriana. Una propuesta desde la regresión cuantílica. Cuestiones económicas, 27(2), 175-177.

Arias-Vargas, M. (2017). Impacto en el inventario de seguridad por la utilización de la desviación estándar de los errores de pronóstico. Tecnología en Marcha, 30(1), 49-54. https://doi. org/10.18845/tm.v30i1.3064

Boada, A. J. (2017). Modelo lineal dinámico bayesiano como procedimiento de actualización automática para modelos estadísticos predictivos. Dimensión Empresarial, 15(1), 33-52. http:// dx.doi.org/10.15665/rde.v15i1.547

Boada, A. J. (marzo de 2017). Sistema de proyección de la demanda. Caso práctico de predicción automatizada en empresas de venta por catálogo. Perspectiva Empresarial, 4(1), 23-41. https://doi.org/10.16967/rpe.v4n1a2

Cadena Lozano, J. B., Ariza Garzón, M. J., \& Palomo Zurdo, R. J. (2017). La gestión de pronósticos en las decisiones empresariales: un análisis empírico. Espacios, 39(13), 1-16.

Contreras Juárez, A., Atziry Zúñiga, C., Martínez Flores, J. L., \& Sánchez Partida, D. (2016). Análisis de series de tiempo en el pronóstico de la demanda de almacenamiento de productos perecederos. Estudios Gerenciales, 32(141), 387-396. https://doi.org/10.1016/j.estger.2016.11.002

Daza Izquierdo, J. (2016). Crecimiento y rentabilidad empresarial en el sector industrial brasileño. Contaduría y Administración, 61(2), 266-282. https://doi.org/10.1016/j.cya.2015.12.001

Delgadillo Ruiz, O., Ramírez Moreno, P. P., Leos Rodríguez, J. A., Salas González, J. M., \& Valdez Cepeda, R. D. (2016). Pronósticos y series de tiempo de rendimientos de granos básicos en México. Acta Universitaria, 26(3), 23-32. http://dx.doi.org/10.15174/au.2016.882

Espino Timón, C. (2017). Análisis predictivo: técnicas y modelos utilizados y

aplicaciones del mismo - herramientas Open Source que permiten su uso (Tesis de pregrado). Universitat Oberta de Catalunya, España. Recuperado de https://bit.ly/31pyZdt

García Guerrero, V. M. (2014). Pronóstico estocástico de la población mexicana utilizando modelos de datos funcionales. Revista Internacional de Estadística y Geografía, 5(3), 18-21.

Garduño García, G. (2011). Metodología para calcular el pronóstico de ventas y una medición de su precisión en una empresa farmacéutica: Caso de estudio (Tesis de pregrado). Instituto Politécnico Nacional, México. Recuperado de https://bit.ly/2MPnubk

Hanke, J. E. (2015). Pronósticos para negocios (novena ed.) México: Pearson Educación.

Herrera, L., Medina, A., \& Naranjo, G. (2010). Tutoría de la Investigación Científica (Cuarta Edición ed.). Quito, Ecuador: Gráficas Corona Quito.

Lamas, C. A. (2016). Los Márgenes de error en las encuestas electorales. Revista Investigación y Marketing (AEDEMO), (132), 1-9.

Lao-León, Y. O., Rivas-Méndez, A., Pérez-Pravia, M. C., \& Marrero Delgado, F. (2017). Procedimiento para el pronóstico de la demanda mediante redes neuronales artificiales. Ciencias Holguín, 23(1), 1-18.

López, F. (2016). La Cuenta de Resultados. Cómo analizarla y gestionarla. Catalunya, España: Libros de Cabecera.

López, P. L. (2014). Población Muestra y Muestreo. Punto Cero, 9(8), 69-74.

M. López, A., A. Flores, M., \& I. Sánchez, J. (mayo de 2017). Modelos de series temporales aplicados a la predicción del tráfico aeroportuario español de pasajeros: Un enfoque agregado y desagregado. Estudios de Economía Aplicada, 35(2), 395-418.

Montañez Muñoz, E. J. (19 de 11 de 2010). Diseño y desarrollo de un modelo de pronóstico para planeación en el área académica de la UNASAM. Revista de investigación Aporte Santiaguino, 3(2), 266-271. http://dx.doi.org/10.32911/as.2010.v3.n2.448

Moreno Arenas, R. (2016). Selección de un método de pronóstico de la Demanda para la estandarización de compras de Empaque y Embalaje. Universidad Militar Nueva Granada, Colombia. Recuperado de https://bit.ly/2YTJDYb 
Moscoso Escobar, J., \& Botero Botero, S. (24 de mayo de 2013). Métodos de Valoración de nuevos Emprendimientos. Semestre Económico, 16(33), 237-262. https://doi.org/10.22395/ seec.v16n33a9

Peña Figueroa, I., \& Paredes Mora, G. (2016). Predicción de la demanda del volumen de madera central en el mercado de Chile para Aserraderos Arauco S.A. Universidad Católica de la Santísima Concepción, Chile. https://doi.org/10.13140/RG.2.2.21899.90404

Render, B., Stair Jr., R. M., \& Hanna, M. (2015). Métodos cuantitativos para los negocios. México: Pearson Educación.

Reyes Sánchez, C. (2017). Marco teórico y empírico para la estimación de sistemas de demanda. Revista Iberoamericana de Producción Académica y Gestión Educativa, 4(7), 1-5.

Romero, L. (2012). Metodología de la investigación en Ciencias Sociales. México: Universidad Juárez Autónoma de Tabasco.

Sánchez, R., \& Gavira Durón, N. (2016). Pronóstico de la demanda de visitantes internacionales: el caso de México. Journal of Research in Accounting and Management Science, 12(23), 29-54. Recuperado de https://bit.ly/31r6pIz

Silva Romero, M. A. (mayo de 2013). Modelo de pronóstico para la estimación de la utilización y confiabilidad de equipos dinámicos. Caso: Equipo de compresión de la RPMNE. Ingeniería Petrolera, 53(5), 287-297. Recuperado de https://bit.ly/2OVTahY

Toca Torres, C. (11 de 10 de 2010). Consideraciones para la Formación en Emprendimiento: Explorando nuevos ámbitos y posibilidades. Estudios Gerenciales, 26(117), 41-60. https://doi. org/10.1016/S0123-5923(10)70133-9

Valencia Cruzaty, L. (17 de Julio de 2017). Emprendimiento en Ecuador. Estudio de Caso: Provincia de Santa Elena. Revista Killkana Sociales, 1(2), 27-32.

Vergara Schmalbach, J. C., Quesada Ibargüen, V. M., \& Maza Ávila, F. J. (2017). Efecto de los aspectos demográficos en la valoración de la calidad del del servicio. Revista U.D.C.A Actualidad E Divulgación Científica, 20(2), 457-467. https://doi.org/10.31910/rudca.v20.n2.2017.401 